\title{
Análisis argumentativo de los debates electorales en la campaña 28-A: la construcción del enemigo
}

\section{Argumentative Analysis of the Electoral Debates in the Campaign 28-A: the Construction of the Enemy}

Miljana Micovic. Centro de Educación Superior Next. España. m.micovic@cesnext.com

$[\underline{\mathrm{CV}}] \odot \mathrm{G}$

Adrià Alsina Leal. Universidad de Vic. Universidad Central de Cataluña. España. adria.alsina@uvic.cat

$[\underline{\mathrm{CV}}]$ (1)

Inmaculada Anaya Revuelta. Universidad de Vigo. España. ianaya@uvigo.es

$[\mathrm{CV}]$ (1) $\mathrm{G}$

Este artículo forma parte de los trabajos desarrollados en el marco del proyecto de investigación "DEBATv, Debates Electorales Televisados en España: Modelos, Proceso, Diagnóstico y Propuesta" (CSO2017-83159$R$ ), proyecto de $I+D+I$ (Retos) financiado por el Ministerio de Ciencia, Innovación y Universidades y la Agencia Estatal de Investigación (AEI) del Gobierno de España, con el apoyo del Fondo Europeo de Desarrollo Regional (FEDER) de la Unión Europea (UE).

Cómo citar este artículo / Referencia normalizada

Micovic, M., Alsina Leal, A. y Revuelta, A. (2020). Análisis argumentativo de los debates electorales en la campaña 28-A: la construcción del enemigo. Revista Latina de Comunicación Social, (76), 189-207. https://www.doi.org/10.4185/RLCS-2020-1443

\section{RESUMEN}

Introducción: La agresividad verbal y la estrategia de ataque han caracterizado los discursos políticos de la campaña electoral para las elecciones generales en España, celebradas el 28 de abril de 2019. Metodología: En este trabajo analizamos los dos debates electorales, celebrados el 22 de abril (RTVE) y el 23 de abril (Atresmedia), desde un punto de vista lingüístico y pragmático. El objetivo es identificar, a través de análisis de discurso, las estrategias argumentativas que utilizan los cuatro líderes de los partidos invitados a los debates para definir y construir al enemigo. Resultados y conclusiones: El análisis de sus intervenciones demuestra que Pedro Sánchez (PSOE), Pablo Casado (PP), Albert Rivera (Ciudadanos) y Pablo Iglesias (Unidas Podemos) coinciden en crear marcos conceptuales y elaborar su argumentación para reforzar la imagen del adversario como enemigo de España. Los candidatos utilizan numerosos recursos lingüísticos y retóricos, y mecanismos pragmáticos de la (des)cortesía lingüística.

PALABRAS CLAVE: discurso político; argumentación; debate; (des)cortesía lingüística. 


\begin{abstract}
Introduction: Verbal aggressiveness and attack strategy towards the rival have been two of the main characteristics of political speeches during the April 2019 Spanish national election campaign. Methodology: This paper analyses the two only TV debates, which were held on April 22 (RTVE) and April 23 (Atresmedia), using a linguistic and pragmatic approach. The objective is to identify, through discourse analysis, the argumentative strategies that the four leaders of the parties invited to use to define and construct the enemy. Results and conclusions: Analysis of their speeches shows that Pedro Sánchez (PSOE), Pablo Casado (PP), Albert Rivera (Ciudadanos) and Pablo Iglesias (Unidas Podemos) create frames and develop their arguments to reinforce the image of their adversaries as enemies of Spain. The four candidates use numerous linguistic and rhetorical resources and pragmatic mechanisms of linguistic (im)politeness.
\end{abstract}

KEYWORDS: political discourse; argumentation; debate; linguistic (im)politeness.

\title{
CONTENIDOS
}

1. Introducción. 1.1. Marco teórico 2. Metodología. 3. Resultados. 3.1. Pedro Sánchez (PSOE). 3.2. Pablo Casado (PP). 3.3. Albert Rivera (C's). 3.4. Pablo Iglesias (Unidas Podemos). 3.5. Análisis comparativo de los resultados. 4. Discusión y conclusiones. 5. Referencias bibliográficas.

\section{Introducción}

El análisis de los debates electorales televisados ha recibido una gran atención desde su aparición. En las últimas décadas, se han realizado numerosos estudios, desde diferentes puntos de vista. El objetivo de esta investigación es llevar a cabo el análisis argumentativo del discurso de los candidatos en los dos debates electorales entre los líderes de los cuatro partidos con mayor representación parlamentaria: Pedro Sánchez del Partido Socialista Obrero Español (PSOE), Pablo Casado del Partido Popular (PP), Albert Rivera de Ciudadanos (C's) y Pablo Iglesias de Unidas Podemos. El primer debate titulado "Debate a cuatro" se celebró el día 22 de abril en RTVE. El moderador fue el periodista de TVE Xabier Fortes. El segundo encuentro, organizado por Atresmedia y titulado "El debate decisivo", el 23 de abril. Los moderadores en esta ocasión fueron los periodistas de Atresmedia Ana Pastor y Vicente Vallés.

En un contexto de gran incertidumbre sobre los resultados de las elecciones, los debates cobraron una gran importancia. El número de indecisos, según el barómetro del Centro de Investigaciones Sociológicas (CIS), publicado el 9 de abril de 2019, ascendía a 41,6\% ${ }^{1}$. Se discute acerca de la repercusión que el debate tiene sobre el electorado $y$, aunque las encuestas en este sentido no son del todo fiables, una buena o mala actuación en un debate está demostrado que puede influir en el voto de los ciudadanos.

Conscientes de esta importancia, los candidatos, además de desmontar el proyecto político del rival, se esfuerzan por dar una imagen negativa del contrario. Para ello, utilizan la agresividad verbal y la estrategia de ataque. Además, la lengua es un instrumento de gran poder que, bien utilizada, puede convertirse en el arma más afilada en la contienda electoral.

\subsection{Marco teórico}

En las últimas décadas, en España han proliferado los estudios de análisis del discurso político en los debates electorales (Fuentes, 2010; Blas Arroyo, 2011; Micovic, 2014; Fernández García, 2017; Anaya y Micovic, 2019, entre otros).

\footnotetext{
1 "El CIS da un cómodo triunfo al PSOE, que dobla al PP en escaños el 28-A", artículo publicado en El País, 9 de abril de 2019, recuperado de: https://elpais.com/politica/2019/04/09/actualidad/1554791390 073854.html
} 
Goffman (1974) introduce el concepto de 'marco' (frame) en las ciencias sociales y de comunicación, investigando las interacciones interpersonales. Según este autor, toda experiencia o actividad social puede contemplarse desde varios "encuadres". Desde la lingüística cognitiva, Lakoff (2004, 2009) reelabora la noción de 'marco' de Goffman y define 'marco conceptual' como un conjunto de "estructuras mentales que dibujan la forma como vemos el mundo"; estas estructuras moldean nuestra visión del mundo, nuestras ideas, acciones y juicios (Lakoff, 2004, p. 15). Los marcos constituyen la base de formación de la opinión pública y, de acuerdo con ello, los políticos intentan imponer la visión de una determinada situación, para lo cual utilizan un lenguaje que transmite ideas favorables a sus posiciones. Al contrario, para referirse al rival, usan un lenguaje impregnado de significado negativo.

En concreto, la campaña del 28-A fue una campaña negativa, es decir, se basó en resaltar los defectos de los adversarios (ideas, posiciones políticas, aspectos de la persona y comportamientos privados), más que en destacar las virtudes de los propios candidatos, partidos, propuestas o posicionamientos políticos (García Beaudoux, D’Adamo y Slavinsky, 2005). Esta estrategia no es nueva. La construcción del enemigo, del "otro" (nosotros vs. ellos) ${ }^{2}$, se remonta a la antigüedad, tal como afirma Fernández Lagunilla (1999, p. 40), "la enunciación política parece inseparable de la construcción de un adversario, tan es así que en política si no existe un adversario hay que fabricarlo".

En cuanto al discurso político actual, Montolío (2019, p. 75) destaca el aumento de la conflictividad verbal y de la argumentación de carácter emocional en las intervenciones de los políticos, señalando que "la agresión verbal al adversario es una falacia para crear ruido con la apariencia de un argumento". Según indica la lingüista, el argumento ad hominem ${ }^{3}$, "es uno de los mejores ejemplos de lo que Cicerón denominó falacias emocionales, desbordamientos pasionales con formato de argumento, pero vacíos de contenido útil para un debate" (Montolío, 2019, p. 75).

Con relación a la tipología argumentativa, este análisis se basa en la teoría de la argumentación de Perelman y Olbrechts-Tyteca (1958). No podemos profundizar en sus esquemas argumentativos, pero sí identificamos algunos de los tipos de argumentos definidos por estos teóricos. Para construir y reforzar la argumentación del rival como enemigo, los líderes políticos utilizan el argumento de superación, la argumentación por analogía o metáfora, la hipérbole, la ironía, la argumentación basada en la disociación de las nociones (pareja filosófica "apariencia-realidad"), el argumento ad personam y la argumentación de carácter emocional.

Asimismo, la elección del léxico con connotaciones negativas para referirse al rival (Morales y Prego, 2002) y el uso del "voto de miedo" tienen como finalidad provocar incertidumbre y temor en los votantes; su objetivo es advertir sobre las consecuencias desastrosas en el caso de que gane el contrario.

Por su parte, Solanilla (2019, p. 7) advierte la presencia de un "lenguaje bélico" y subraya que estos dos debates fueron "un espectáculo decepcionante de confrontación de ideas, proyectos y personas".

Para el comentario pragmático del análisis de los debates objeto de estudio de este trabajo, partimos del esquema diseñado por Cortés $(1991,2008)^{4}$, según el cual hay dos niveles: “el nivel de variación

\footnotetext{
${ }^{2}$ La construcción del "otro" se ha estudiado desde la perspectiva de Análisis Crítico de Discurso (ACD) (Fairclough, 1995, Wodak, 2000; Van Dijk, 2005, 2008, entre otros) en la que se investiga la relación entre el discurso y la ideología.

${ }^{3}$ En la Teoría de la argumentación, Perelman y Olbrechts-Tyteca (1958) denominan este tipo de argumento argumento ad personam. En el presente estudio, usamos esta denominación.

${ }^{4}$ En el nivel de variación interna, Luis Cortés (2008) incluye todo lo relacionado con las formas y mecanismos lingüísticos encaminados a conseguir los efectos discursivos que pretende el hablante. Él explica que se le planteaban dos opciones de estudio: separar los apartados por temas y analizar el texto en bloque; o segmentar el texto en tres partes y analizar cómo se presentan los temas en cada una de ellas. En este trabajo solo señalamos algunos de los recursos lingüísticos y pragmáticos empleados en los dos debates.
} 
externa o condicionantes contextuales" y "el nivel de variación interna o condicionantes semiolingüísticos", que es el que nos ha servido para comentar algunos aspectos del nivel lingüístico, como el uso de los pronombres personales, la presencia de marcadores del discurso, etc. En el nivel de mecanismos discursivos, destacamos algunos como el uso de la enumeración, la estructura de las frases y el empleo de la metáfora.

También desde la perspectiva pragmática, estudiamos el uso de las estrategias de (des)cortesía con función argumentativa. Dentro del tono formal que exigen los debates, en los celebrados en esta campaña, se desarrolla un particular comportamiento de agresividad verbal, en el que el desacuerdo y la crítica están permanentemente presentes (Fernández García, 2017) ${ }^{5}$. Entre los mecanismos empleados, la acusación de mentir es uno de los utilizados con mayor frecuencia y contribuye a la creación de la imagen del oponente como enemigo del país, una de las agresiones más duras contra la imagen del rival.

\section{Metodología}

Para llevar a cabo el análisis argumentativo del discurso de los candidatos en los dos debates electorales, hemos utilizado el corpus audiovisual conformado por el "Debate a cuatro" (RTVE), del 22 de abril de 2019, y "El debate decisivo" (Atresmedia), del 23 de abril de 2019. Los vídeos de ambos debates están disponibles íntegramente en internet ${ }^{6}$.

En el caso del debate en la RTVE, existe también la transcripción íntegra del debate en la misma página web en la que se encuentra el vídeo. Sin embargo, el debate celebrado en Atresmedia no dispone de una transcripción, por lo que ha sido necesario realizar la transcripción correspondiente a las intervenciones de cada candidato, consideradas relevantes para este estudio. Los ejemplos del primer debate aportados en el análisis en el epígrafe 3 se indican con (D-1), y los del segundo, con (D-2).

En primer lugar, para identificar los argumentos y los recursos lingüísticos utilizados en el debate con el objetivo de construir al enemigo, se han visualizado los dos debates en su totalidad y se han seleccionado todas las intervenciones de los cuatro candidatos en las que se argumenta que al adversario político es enemigo de España y de los españoles.

El análisis realizado ha constado de tres fases:

A. Primero, hemos identificado y hemos descrito los marcos cognitivos establecidos por cada candidato. El objetivo de esta fase ha sido comprobar que cada uno de los marcos corresponde a la construcción de un enemigo en concreto. La formulación de los marcos no está extraída literalmente del discurso de los candidatos, sino que hemos resumido los marcos principales para abarcar un amplio abanico de argumentos usados para sustentarlos. El número máximo de marcos construidos por los candidatos ha sido tres.

B. En segundo lugar, se han identificado los argumentos usados por cada uno de los candidatos que les sirven para construir al enemigo y se han distribuido estos argumentos dentro de cada uno de los marcos establecidos. El objetivo de esta fase ha sido determinar la tipología de los argumentos utilizados.

\footnotetext{
${ }^{5}$ Según la Teoría Funcional de Benoit (2002), los ataques o críticas al oponente (attacks) son una de las tres funciones básicas que usan los candidatos en los debates para evidenciar las limitaciones o debilidades del oponente. Fernández García (2017) también indica que dos funciones discursivas esenciales del debate político son el ataque y la defensa.

${ }^{6}$ El debate de RTVE, disponible aquí: http://www.rtve.es/alacarta/videos/especiales-informativos/especial-informativodebate-cuatro/5159816/

El debate de Atresmedia, disponible aquí: https:/www.atresplayer.com/noticias/el-debate-de-atresmedia/2019/23a-eldebate-decisivo 5cbf7c277ed1a8b0f55ba826/
} 
C. Por último, a través de un análisis detallado, se han reconocido los recursos lingüísticos y retóricos, así como los mecanismos pragmáticos que utilizan los políticos para persuadir al electorado de que su rival es una amenaza para el país y para los ciudadanos. El objetivo de esta fase ha sido, sobre todo, identificar los recursos y mecanismos con función argumentativa más recurrentes en el discurso de cada candidato. Teniendo en cuenta el significado que estos elementos tienen desde un punto de vista teórico (por ejemplo, el significado que los distintos estudiosos de la materia han dado a los marcadores del discurso), analizamos el valor de cada elemento, partiendo del significado que tienen en el contexto en que aparecen, independientemente de lo que signifiquen desde un punto de vista teórico.

Los ejemplos, muy numerosos en este trabajo, son la base de la que parte todo el estudio. Esta metodología nos permite llegar, en nuestra opinión, a conclusiones fidedignas sobre el comportamiento de los candidatos, ya que, a partir de sus intervenciones -los ejemplos- construimos lo que podríamos calificar como el argumentario y el resultado de las distintas posturas.

\section{Resultados}

Desde sus primeras intervenciones, los líderes de los cuatro partidos empiezan a construir al enemigo, a delimitar de manera clara a sus adversarios. Con este objetivo se centran en definirse y diferenciarse del rival. A continuación, analizamos cómo cada uno de los candidatos dirige sus críticas hacia los otros y qué recursos lingüísticos utiliza para llevar a cabo su argumentación.

\subsection{Pedro Sánchez (PSOE)}

El enemigo principal en su discurso es la derecha. Este sustantivo se refiere, en ocasiones, a los gobiernos anteriores del Partido Popular, a los tres partidos que él presenta como un bloque de la derecha, PP, C's y VOX, o a los dos partidos cuyos representantes participan en el debate (PP y C's). Para dirigirse al partido VOX, Sánchez usa el sustantivo ultraderecha, evitando así mencionar el nombre del partido.

Uno de los marcos que crea Sánchez es: "La derecha es moralmente mala e impide el desarrollo del país". En el ejemplo (1), este candidato usa la ironía (pequeños detalles, malvados socialistas) para deslegitimar la gestión del anterior gobierno del PP. Utiliza la argumentación de disociación de las nociones para hablar de las dos historias, la del PP y la del PSOE, mediante la cual Sánchez pretende convencer al público de que él es quien conoce la realidad del país, y sus rivales están alejados de ella:

(1) En este debate ustedes van a escuchar dos historias $^{7}$, una es la que cuenta a diario la derecha; según ellos, España iba maravillosamente bien hasta que los malvados socialistas presentaron una moción de censura. (...) Es verdad que había algunos detalles que se pasaban por alto, por ejemplo, dos referéndums ilegales, (...) una sentencia demoledora contra la corrupción del Partido Popular, pero, salvo estos pequeños detalles, para la derecha española todo iba maravillosamente bien en nuestro país. (D-1)

\footnotetext{
${ }^{7}$ La cursiva se usará en los ejemplos para destacar las estrategias y mecanismos comentados en el análisis.
} 
La forma de comenzar su intervención, anunciando "las dos historias" de España, es un ejemplo de cómo Sánchez emplea, para conseguir diferenciarse del partido de la oposición (el PP), uno de los mecanismos discursivos más efectivos: la enumeración, operación textual mediante la cual el candidato presenta su intervención como un todo coherente. Es discutible si es lo más adecuado o no comenzar una intervención, como la de Pedro Sánchez en este debate, con una enumeración, ya que puede restar algo de emoción a la exposición; sin embargo, en este caso, la división en dos historias ayuda a los interlocutores a entender la dicotomía entre unos y otros, y a recordar el mensaje.

La argumentación que elabora Sánchez sobre la corrupción del PP y su falta de credibilidad pertenecen al mismo marco. En los ejemplos (2) y (3), el político usa la metáfora para desacreditar al partido rival:

(2) La sede del Partido Popular en la calle Génova de Madrid era como el gran bazar de la corrupción. Uno iba a la primera planta, financiación irregular. Segunda planta, enriquecimiento ilícito. Tercera planta, reparto de sobres con dinero en B o sobresueldos. En la cuarta planta, coordinando, la policía patriótica; y en la quinta planta, la agencia Pasadena, liderada por Francisco Correa, el líder de la trama Gürtel. (D-1)

(3) (...) pero si nos vamos a la comunidad valenciana (...) hay tres expresidentes de la Comunidad Valenciana o procesados o imputados o involucrados en casos de corrupción; trece consejeros, es decir, allí en Valencia, no es que hubiera un gran bazar de corrupción, lo que había era un parque temático de la corrupción. (D-2)

Dentro del mismo marco, Sánchez refuerza la idea con el argumento de superación, indicando que la oposición, formada por el PP y C's, es un obstáculo para la prosperidad del país:

(4) Esto lo hemos hecho, desgraciadamente, con la oposición de las dos derechas, aquí presentes, con bloqueos parlamentarios que han impedido el debate y la aprobación de iniciativas parlamentarias para la mayoría social de este país. (D-1)

En otros momentos, la crítica va dirigida a los políticos, a las personas y a sus comportamientos. Esta crítica se refuerza con otro de los mecanismos lingüísticos más empleados por los cuatro candidatos: el uso de conectores discursivos, como claro y además (5). El conector claro refuerza el sentido argumentativo de lo dicho anteriormente; el conector además denota que los argumentos no se ordenan de una manera arbitraria (Montolío, 2001), ya que el argumento que sigue a este marcador destaca, generalmente, la idea considerada más relevante para transmitir el mensaje.

(5) Claro, uno ve las propuestas de revolución fiscal que dice el señor Casado o la derecha y, claro, las ve profundamente injustas y, además, profundamente irresponsables. (D-1) 
En otros ejemplos, además del uso de conectores como bueno, en este caso con valor conclusivo, se utilizan también recursos retóricos, como los juegos de palabras, basados en la oposición de contrarios (verdades/mentiras):

(6) Bueno, uno ya está acostumbrado a estas mentiras, a las falsedades, a las descalificaciones

hiperbólicas de la derecha, del señor Casado, del señor Rivera. En lugar de un detector de mentiras, habrá que poner un detector de verdades, a ver si dicen alguna verdad. (D-1)

(7) Aquí las mentiras vuelan entre el Sr. Casado y Sr. Rivera y aquí son indistintas, (...) cada vez se parecen más y cada vez se parecen más a la ultraderecha. (D-2)

(8) Yo no he pactado con los independentistas, nunca. Que es falso (...) y, por tanto, usted [Rivera] podrá repetir mil veces una mentira, pero es falso. Falso es falso. No es no y nunca es nunca. (D-2)

En los ejemplos (6), (7) y (8) se han señalado en cursiva también las palabras mentira, falsedad, falso dado que Sánchez recurre a la estrategia de descortesía de acusación de mentir para reforzar su argumentación. La mentira se presenta como una característica propia y común al enemigo, formulado como derecha, ultraderecha, o bien, de manera personalizada en los candidatos. Además, en el ejemplo (8) observamos el uso de tautología (no es no, falso es falso), muy recurrente en el discurso político, como también señala Montolío (2019).

Sánchez agrupa a los tres partidos de la derecha dentro de otro marco: "La ultraderecha es un gran peligro para España y, si suma con las otras dos derechas, van a gobernar juntos". En el ejemplo (9) cabe señalar otro concepto que usa Sánchez para designar al enemigo: el trío de Colón ${ }^{8}$. Además, en los ejemplos (10) y (11), este político usa la estrategia del "voto del miedo", al señalar el riesgo que supone que ganen los tres partidos de la derecha. En el nivel lingüístico, el uso reiterado de la forma yo (10), frente a la forma uno, en tercera persona, utilizada en el ejemplo (6), personaliza el mensaje, le distancia del "otro" (el rival) y le confiere cierto sentido de autoridad y de credibilidad (Leanne, 2009):

(9) El próximo 28 de abril los españoles estamos convocados a decidir qué país queremos, si queremos una España en la que quepamos todos, todas, o una España en la que solamente cabe el trío de Colón. (D-2)

(10) (...) van a tener al señor Casado de presidente, al señor Rivera de acompañante en algún ministerio y la ultraderecha a los mandos. Y, fijense, yo creo que esta es una realidad muy peligrosa que tenemos que evitar, (...) yo creía que en Andalucía no iban a pactar, Ciudadanos, el Partido Popular y la ultraderecha, y han pactado. (D-1)

\footnotetext{
${ }^{8}$ El concepto proviene de la manifestación en la Plaza de Colón de Madrid, el día 10 de febrero de 2019, convocada por los tres partidos como protesta contra Sánchez, sus negociaciones con los independentistas y para apoyar la unidad de España.
} 
(11) Hay un riesgo de que sumen estos dos [Rivera y Casado] con la ultraderecha ausente. La ultraderecha es peligrosa, es temible, dice cosas en relación con este asunto: "Una mujer violada no tiene derecho a abortar, "los colectivos LGTBI generan cada vez más niños que pertenecen a ese colectivo. Que habla de "las mujeres piojosas de la ultraizquierda" y que considera que la verdadera dictadura no ha sido la franquista, sino la feminista. (D-2)

Por último, Sánchez crea un marco para situar al independentismo catalán como una amenaza para los ciudadanos españoles, al mismo nivel que la derecha. Así, el ejemplo (12) refuerza la idea de que el independentismo, pero también todos los partidos, excepto el PSOE y Unidas Podemos, son enemigos de los españoles:

(12) Estamos en elecciones anticipadas el 28 de abril porque el independentismo, el PP y

Ciudadanos votaron en contra de unos presupuestos que habíamos pactado Unidas Podemos y

nosotros. (D-2)

En cuanto a Unidas Podemos, Sánchez no dirige ninguna crítica hacía esta formación; más bien, hay un agradecimiento explícito por el apoyo a sus políticas:

(13) $\mathrm{Y}$ en esto quiero ser claro y agradecerlo, Unidas Podemos y el señor Iglesias lo han

apoyado y yo quiero darles las gracias públicamente. (D-1)

\subsection{Pablo Casado (PP)}

El primer marco que crea Casado es: "Pedro Sánchez es un traidor por su asociación con independentistas y etarras". En él, el líder del PP relaciona la presunta violencia del "golpe de Estado" independentista con la violencia terrorista de ETA. En los ejemplos seleccionados, Casado formula argumentos que sustentan estos marcos; se trata de una serie de críticas a Sánchez, dado que su objetivo es plantear la contienda electoral entre su proyecto (la única alternativa) y el de Sánchez. En su interés por reforzar sus ideas, emplea la repetición de estructuras sintácticas, precedidas en este caso por el adverbio quizá, que atenúa la afirmación y le confiere un tono moderado a sus palabras:

(14) Hace nueve meses había un gobierno en España del PP (...) que había llevado a los

golpistas de Puigdemont al banquillo (...) Quizá por eso, el señor Torra y el señor Otegi apoyaron la moción de censura que presentó Pedro Sánchez, y quizá por ello hayan dicho esta semana que le volverían a apoyar, si suman, después de estas elecciones generales. (...) [el $\mathrm{PP}$ ] es la única alternativa presente en este plató. (D-1)

(15) La unidad de España está en riesgo por culpa del gobierno socialista de Pedro Sánchez; es muy sencillo de decir, los que quieren romper a España tienen a Sánchez como su candidato favorito. (D-1) 
Casado hace uso de la metáfora en varias ocasiones. En el ejemplo (16), Sánchez es una matrioska, una muñeca rusa que contiene otras idénticas y -esto es lo interesante en la asociación de ideas- de menor tamaño. Cuanto más original y acertada sea esta asociación, mayor será el impacto en el receptor y mayor, por tanto, su perdurabilidad en la mente del que la escucha. Así, según esta metáfora, Sánchez engloba a Podemos, a Esquerra Republicana, a JuntsxCat e, incluso, a Bildu:

(16) El problema es que el Sr. Sánchez se ha convertido en una muñeca rusa, una matrioska, dentro de él está Podemos, está Esquerra Republicana, está JuntsxCat y está, también, Bildu.

El líder del PP acusa a Sánchez de ser un traidor, incluso, con su propio partido (17), y utiliza para ello la acusación de mentir (18):

(17) Que los va a indultar, que ya tiene la hoja de ruta, que quiere volver a revalidar ese

Gobierno Frankenstein, como dice Rubalcaba, con independentistas, batasunos, con los de

Podemos y, sobre todo, traicionando a la historia democrática del PSOE. (D-1)

(18) Yo es que ahora recuerdo a Felipe González cuando decía en una entrevista: "Es que con

Pedro Sánchez te está diciendo una cosa y sabes que te está mintiendo al mismo tiempo”. (D-1)

Pero los ataques personalizados a Sánchez no solo se formulan con respecto a la unidad de España, sino también con relación a sus políticas, como en el ejemplo (19), en el que Casado usa la ironía:

(19) Cada vez que abre la boca el Sr. Sánchez sube el pan, el diesel y la luz. Los españoles

estamos en deuda con el Sr. Sánchez. (D-2)

El líder del PP usa el argumento ad personam y la hipérbole, dirigida a cuestionar la idoneidad de Sánchez como candidato a la presidencia de Gobierno:

(20) Sinceramente, es el colmo de la humillación y usted, sinceramente, señor Sánchez, no da

la talla como presidente del Gobierno. (D-1)

(21) Es usted el presidente más radical de toda la democracia. (D-2)

(22) Usted ahora mismo es un peligro público para España. (D-2)

El siguiente marco que crea Casado ataca no al candidato Sánchez, sino a su partido: "El socialismo y la izquierda son enemigos de España":

(23) Yo me quiero dirigir a esos españoles que recuerdan en carne propia y muy recientemente, cómo en el gobierno del señor Rodríguez Zapatero, 3,5 millones se fueron a la calle, (...) con el voto del diputado Sánchez, también, (...) para sufragar también los servicios sociales que otros han puesto en riesgo, no solo en el actual gobierno, sino también en el anterior de Zapatero, y en el de los años 90, del señor González. (D-1) 
(24) (...) el gasto social, que con el gobierno del Partido Popular incrementó 26.000 millones de euros para empezar, por los estragos que causó la crisis económica de la izquierda. (D-1)

(25) El Partido Socialista nunca ha creado empleo, el Sr. Felipe González desde el año 82 hasta el año 96 creó 0 empleos netos. (D-2)

Por último, este candidato declara también quién no es su enemigo, tal como puede verse en la siguiente intervención, contestada por Rivera:

(26) Casado: Yo creo, señor Rivera, que ni sus votantes ni los míos entienden sus acusaciones

y sus ataques a mi persona (...) porque usted no es mi adversario.

- Rivera: Ni usted tampoco. (D-1)

\subsection{Albert Rivera (C's)}

El principal marco que crea Rivera coincide con el primer marco creado por Casado: "Pedro Sánchez es un traidor porque es cómplice de los nacionalistas y separatistas". Dentro del marco, también define al independentismo catalán como "golpistas". En su primera intervención, el líder de Ciudadanos ataca de forma agresiva y lanza reproches muy graves a Sánchez, que abarcan varias áreas de su mandato; concluye con la crítica a su gestión sobre la cuestión catalana:

(27) El señor Sánchez no se conforma con colocar a dedo a la presidenta de Televisión

Española; lo ha hecho en Correos, lo ha hecho manipulando el CIS, lo ha hecho colocando amigos y enchufados en empresas públicas, hasta 500 cargos. Pero, además, ahora quiere indultar; lleva en la frente la palabra "indulto" para indultar a sus socios separatistas que han dado un golpe de Estado. (D-1)

En ambos debates, Rivera formula argumentos dentro de dicho marco. En el ejemplo (28), este político usa la hipérbole, en (29) y (30) recurre a la argumentación de carácter emocional, a la metáfora (silencio) y a la repetición; en (31) utiliza la acusación de mentir. Utiliza con frecuencia dos verbos con una fuerte carga connotativa, romper ('separar con violencia', 'destrozar') y un sinónimo de este, liquidar.

(28) Usted es el máximo defensor de las políticas de los nacionalistas, por eso les representa usted aquí en este debate. (D-1)

(29) A mí me duele España, a mí me duele que se rompa Cataluña, porque además soy catalán. Al señor Sánchez no le importa, el señor Sánchez es capaz de sentarse con el señor Torra y hacerse la foto de 21 condiciones de la vergüenza, poner un mediador y lo que haga falta para seguir en el poder. (D-1) 
(30) ¿Lo escuchan? Es el silencio, el silencio que nos heló la sangre a millones de españoles cuando los separatistas quisieron romper nuestro país en Cataluña, [...) Es el silencio cómplice de Pedro Sánchez. (D-1)

(31) El Sr. Sánchez ha mentido, ha dicho que no ha pactado con los separatistas. (D-2)

Rivera expresa que "los cómplices" de Sánchez son, también, Bildu y su líder; utiliza, para ello un léxico con fuerte carga semántica de connotación negativa:

(32) El Sr. Otegui ha ofrecido un millón de votos para votar al Sr. Sánchez. Sánchez es el candidato del terrorista Otegui, del de Bildu. (...) quiere pactar con los que quieren liquidar España. (D-2)

Mediante el uso de la metáfora en el ejemplo (33), Rivera señala que Unidas Podemos son socios de Sánchez y advierte de los daños que puede ocasionar esta coalición a la economía española:

(33) Si viene el señor Iglesias de ministro y vicepresidente del señor Sánchez, cójanse la cartera porque van a ir a saco. (...) es que le van a meter la mano en la cartera a los ciudadanos. (D-1)

Frente a todos estos "enemigos de España" y "la traición de Sánchez", el candidato de Ciudadanos introduce el concepto de emergencia nacional con el que pretende reforzar su argumentación principal sobre la gravedad de la situación para el país. Esta idea la repite en ambos debates:

(34) Es una emergencia nacional enviar al señor Sánchez, a los separatistas y al señor Iglesias

a la oposición y formar un gobierno constitucionalista (...) que es una emergencia nacional enviarle a la oposición. (D-1)

Entre los ataques a Sánchez, están los formulados por Rivera como crítica personal, a través del argumento ad personam y la acusación de mentir:

(35) Señor Sánchez, que usted es el de la tesis de mentira, no diga..., no hable de mentiras,

que tiene una tesis de mentira, que le han pillado. (D-1)

El líder de Ciudadanos, en ocasiones, traslada su crítica a todo el Partido Socialista:

(36) El Partido Socialista, quién lo ha visto y quién lo ve, un partido que pedía igualdad en los

años 80 y que hoy es el utensilio de los nacionalistas. (D-1)

En este sentido, para criticar a los partidos tradicionales y al bipartidismo, Rivera establece otro marco: "Los viejos partidos (PSOE y PP) no han hecho (absolutamente) nada en los pasados 40 años". Para designar al enemigo, en este caso, utiliza la fórmula de azules y rojos, o (la vieja) izquierda y derecha, entre otras: 
(37) La realidad es que escucho los discursos de la vieja izquierda y derecha y sigue en el siglo pasado, izquierda y derecha peleándose, pero sin propuestas. (D-1)

(38) (...) para acabar con este debate de "y tú más” de azules y rojos, y rojos y azules, que no nos lleva a ningún sitio. (D-2)

(39) El PP y el PSOE se han pasado 40 años sin pactar la educación. (...) sin hacer nada salvo llevarnos al fracaso. (D-2)

Finalmente, aun cuando afirma que el líder del PP no es su adversario, con el objetivo de diferenciarse de él, Rivera formula algunas críticas y sitúa el partido rival dentro del marco de los viejos partidos que enfrentan un problema muy grave, la corrupción:

(40) ¿[Sr. Casado] Sabe dónde está el milagro económico del Partido Popular? En la cárcel.

Aun así, el candidato de C's deja claro que el PP es un posible aliado, y por tanto, no es su enemigo:

(41) Tiendo la mano al PP porque, insisto, creo que somos dos partidos que podemos formar

la coalición de gobierno. (D-2)

\subsection{Pablo Iglesias (Unidas Podemos)}

La argumentación en el discurso de Iglesias, en los dos debates, se basa en un marco en el que también hay "buenos" y "malos" enfrentados: los "malos" son los de "arriba" (los poderes económicos y políticos) y los "buenos" los de "abajo" (los ciudadanos), defendidos por él y por su partido.

Iglesias no define a los rivales políticos presentes en el plató de televisión como enemigos, sino que describe un conflicto que va más allá creando el siguiente marco: "La minoría privilegiada y corrupta de grandes poderes económicos aprovecha sus vínculos con el gobierno para mantener privilegios". Por tanto, Iglesias construye un enemigo poderoso frente a unos ciudadanos desprotegidos y presenta a los partidos políticos de sus adversarios como defensores de esa minoría privilegiada a través de la corrupción ${ }^{9}$.

En los ejemplos (42), (43) y (44) Iglesias defiende que esa minoría está compuesta por los bancos, las grandes multinacionales, los fondos buitre, las empresas energéticas y los medios de comunicación que, según él, están al servicio de los privilegiados. En sus argumentaciones usa diferentes recursos encaminados a conseguir una mayor fuerza expresiva: las repeticiones, las palabras negativas, la metáfora, etc.

(42) Decimos que es una vergüenza que los bancos no paguen en la práctica Impuesto de

Sociedades en este país; (...) no puede ser que siempre la gente trabajadora sea la que paga impuestos y que haya una minoría de privilegiados que no hacen ese esfuerzo. (D-1)

\footnotetext{
9 El "antielitismo" en el discurso político es una de las principales características del discurso populista, según Reinemann et al. (2016). Arroyas y Pérez (2016), entre otros, estudian el populismo en el discurso de Pablo Iglesias.
} 
(43) Es legal que los ministros y expresidentes acaben en consejos de administración de empresas energéticas, pero eso es corrupción. Esas empresas están comprando políticos. (D-2)

(44) Es verdad que los dueños de los medios de comunicación tienen más poder que los diputados. Es verdad que los bancos de España doblaron el brazo al Tribunal Supremo. Es verdad que las empresas energéticas se compran los políticos llevándolos a consejos de administración y es verdad que las cloacas $^{10}$ seguirán trabajando para que no estemos en un gobierno. (D-2)

En el ejemplo (45) Iglesias afirma que el problema de la política en España es la corrupción; puntualiza que el "verdadero enemigo" es la corrupción:

(45) Se ha utilizado una expresión muy fuerte: los enemigos de España. Los enemigos de

España son los que privatizan el patrimonio público, los enemigos de España son los corruptos. (D-2)

Otro marco que crea Iglesias en los debates es el de que "Los viejos partidos, el PP y el PSOE, no cumplen con lo que prometen":

(46) Este artículo de la Constitución no se cumple, y para que se cumpla hay que hacer cosas muy sencillas, la primera, acabar con la estafa de la temporalidad. (D-1)

Por otra parte, Iglesias advierte a Sánchez que no cumplió con lo que prometió en sus acuerdos previos:

(47) Le tengo que recordar que algunas cosas que ustedes se comprometieron a hacer no las hicieron; ustedes no publicaron la lista de amnistiados fiscalmente (...). (D-1)

Con respecto a los pactos poselectorales, Iglesias ve posible pactar con el PSOE, pero no, si estos pactan con Ciudadanos. En este sentido, muestra sus dudas ante la falta de respuesta de Sánchez a esta cuestión:

(48) Pero me gustaría que usted aclarara de una vez a sus electores si usted está dispuesto a llevar a cabo un acuerdo con Ciudadanos. (D-1)

Finalmente, Iglesias plantea un "reenmarcado" (Lakoff, 2004) acerca de cómo se define España, reinterpretando un concepto ajeno y cambiando el marco defendido por otros partidos:

(49) Creo que hay algunos que se envuelven en la bandera España a los que les gusta España, porque España no solo es lo que vimos en la Plaza de Colón, que es muy respetable. Pero España

\footnotetext{
${ }^{10}$ El uso de la metáfora de cloacas para designar las supuestas prácticas ilegales del Ministerio de Interior y del Estado español ha entrado en uso después de la emisión del documental Las cloacas de Interior, del año 2017.
} 
son más cosas. A muchos españoles no les gustan las corridas de toros, muchos españoles se identifican con símbolos diferentes. Muchos españoles hablan lenguas diferentes (...). (D-2)

\subsection{Análisis comparativo de los resultados}

Los resultados obtenidos en el análisis comparativo ponen de manifiesto que los cuatro candidatos utilizan recursos muy parecidos en la elaboración de su discurso; estos recursos están encaminados a atacar al enemigo y a intentar sorprender al público. En la tabla 1 aparecen los rasgos más caracterizadores de la forma de intervenir cada uno de los candidatos desde el punto de vista del análisis del discurso. A diferencia de la forma de debatir en otros momentos en España o, incluso, a diferencia de cómo se debate hoy en otros lugares del mundo, el objetivo fundamental en caso de los cuatro políticos es minar al otro y conseguir emocionar al votante. Podemos observar en el análisis que ciertos tipos de argumentos, recursos retóricos, lingüísticos y pragmáticos se repiten en caso de diferentes candidatos y son frecuentes en los discursos de los debates electorales, así como en el discurso político, en general.

Tabla 1. Estrategias argumentativas, mecanismos discursivos, recursos retóricos y lingüisticos usados por cada candidato.

\begin{tabular}{|c|c|c|c|c|}
\hline & $\begin{array}{l}\text { Pedro Sánchez } \\
\text { (PSOE) }\end{array}$ & $\begin{array}{l}\text { Pablo Casado } \\
\text { (PP) }\end{array}$ & $\begin{array}{l}\text { Albert Rivera } \\
\text { (C's) }\end{array}$ & $\begin{array}{c}\text { Pablo Iglesias } \\
\text { (Unidas Podemos) }\end{array}$ \\
\hline Argumentos & $\begin{array}{l}\text { Argumentación de } \\
\text { la disociación de las } \\
\text { nociones para hablar } \\
\text { de dos historias } \\
\text { Argumentación de } \\
\text { la superación } \\
\text { "Voto del miedo" }\end{array}$ & $\begin{array}{l}\text { Argumentación } \\
\text { ad personam } \\
\text { Ataques } \\
\text { agresivos a } \\
\text { Sánchez }\end{array}$ & $\begin{array}{l}\text { Argumentación de } \\
\text { carácter emocional } \\
\text { Argumentación ad } \\
\text { personam } \\
\text { Ataques agresivos a } \\
\text { Sánchez }\end{array}$ & $\begin{array}{l}\text { Argumentación de } \\
\text { contrarios }\end{array}$ \\
\hline $\begin{array}{l}\text { Mecanismos } \\
\text { discursivos }\end{array}$ & $\begin{array}{l}\text { La enumeración } \\
\text { Uso de conectores } \\
\text { discursivos como } \\
\text { claro, además y } \\
\text { bueno } \\
\text { Acusación de mentir }\end{array}$ & $\begin{array}{l}\text { Acusación de } \\
\text { mentir } \\
\text { Marcadores } \\
\text { discursivos, } \\
\text { como el } \\
\text { atenuante quizá }\end{array}$ & $\begin{array}{l}\text { Acusación de } \\
\text { mentir }\end{array}$ & $\begin{array}{l}\text { Uso de marcadores } \\
\text { encaminados a poner de } \\
\text { relieve los incumplimientos } \\
\text { por parte de Sánchez, como } \\
\text { el conector pero }\end{array}$ \\
\hline $\begin{array}{l}\text { Recursos } \\
\text { retóricos }\end{array}$ & $\begin{array}{l}\text { Ironía } \\
\text { Metáfora } \\
\text { Oposición de } \\
\text { contrarios } \\
\text { Tautología } \\
\end{array}$ & $\begin{array}{l}\text { Metáfora } \\
\text { Ironía } \\
\text { Hipérbole }\end{array}$ & $\begin{array}{l}\text { Hipérbole } \\
\text { Metáfora } \\
\text { Repetición de } \\
\text { palabras }\end{array}$ & $\begin{array}{l}\text { Metáfora } \\
\text { Repetición de estructuras } \\
\text { sintácticas al comienzo de } \\
\text { las frases }\end{array}$ \\
\hline $\begin{array}{l}\text { Recursos } \\
\text { lingüísticos }\end{array}$ & $\begin{array}{l}\text { Uso del pronombre } \\
\text { personal yo para } \\
\text { personalizar el } \\
\text { mensaje frente al } \\
\text { uso de uno en } 3^{\mathrm{a}} \\
\text { persona del singular } \\
\text { Orden de las } \\
\text { palabras alterado } \\
\text { para conseguir el } \\
\text { énfasis } \\
\text { Expresiones } \\
\text { coloquiales }\end{array}$ & $\begin{array}{l}\text { Repetición de } \\
\text { estructuras } \\
\text { sintácticas }\end{array}$ & $\begin{array}{l}\text { Uso de léxico con } \\
\text { una gran carga } \\
\text { semántica negativa: } \\
\text { romper, liquidar, } \\
\text { terrorista. } \\
\text { Abundantes } \\
\text { expresiones } \\
\text { coloquiales: y tú } \\
\text { más, quien lo ha } \\
\text { visto y quién lo ve, } \\
\text { etc. } \\
\text { Contraposición de } \\
\text { los conceptos } \\
\text { azules y rojos / } \\
\text { rojos y azules. }\end{array}$ & $\begin{array}{l}\text { Uso frecuente de palabras } \\
\text { negativas para describir la } \\
\text { realidad } \\
\text { Repetición de estructuras } \\
\text { sintácticas para enfatizar } \\
\text { sobre su mensaje }\end{array}$ \\
\hline
\end{tabular}

Fuente: elaboración propia. 
Asimismo, en la tabla 2 ofrecemos un resumen de los principales marcos usados por cada candidato, así como de los enemigos que cada uno construye en su discurso. Puede observarse que, en cuanto a la construcción del enemigo, hay ciertas coincidencias entre distintos candidatos. En primer lugar, tanto Casado como Rivera identifican como enemigo a Pedro Sánchez, junto con los nacionalistas e independentistas ("golpistas"). Formulan para ello los principales marcos casi idénticos. Por otro lado, Rivera e Iglesias, como líderes de los "nuevos partidos", aluden a los "viejos partidos" como enemigos de España por su mala gestión en el pasado.

Tabla 2. Resumen de los marcos y enemigos que construye cada candidato.

\begin{tabular}{|c|c|c|c|c|c|c|c|}
\hline \multicolumn{2}{|c|}{$\begin{array}{l}\text { Pedro Sánchez } \\
\text { (PSOE) }\end{array}$} & \multicolumn{2}{|c|}{$\begin{array}{c}\text { Pablo Casado } \\
\text { (PP) }\end{array}$} & \multicolumn{2}{|c|}{$\begin{array}{c}\text { Albert Rivera } \\
\text { (C's) }\end{array}$} & \multicolumn{2}{|c|}{$\begin{array}{c}\text { Pablo Iglesias } \\
\text { (Unidas Podemos) }\end{array}$} \\
\hline Enemigo & $\begin{array}{l}\text { La derecha } \\
\text { La ultraderecha } \\
\text { Independentismo }\end{array}$ & Enemigo & $\begin{array}{l}\text { Pedro Sánchez } \\
\text { Nacionalistas / } \\
\text { independentistas } \\
\text { /golpistas / } \\
\text { Podemos } \\
\text { La izquierda }\end{array}$ & Enemigo & $\begin{array}{l}\text { Pedro Sánchez } \\
\begin{array}{l}\text { Nacionalistas / } \\
\text { independentistas } \\
\text { / golpistas }\end{array} \\
\begin{array}{l}\text { Los viejos } \\
\text { partidos }\end{array}\end{array}$ & Enemigo & $\begin{array}{l}\text { Los de } \\
\text { arriba / los } \\
\text { privilegiados } \\
\text { / los } \\
\text { corruptos } \\
\text { Los viejos } \\
\text { partidos }\end{array}$ \\
\hline Marco & $\begin{array}{l}\text { "La derecha es } \\
\text { moralmente } \\
\text { mala e impide el } \\
\text { desarrollo del } \\
\text { país". } \\
\text { "La ultraderecha } \\
\text { es un gran } \\
\text { peligro para } \\
\text { España y, si } \\
\text { suma con las } \\
\text { otras dos } \\
\text { derechas, van a } \\
\text { gobernar } \\
\text { juntos". } \\
\text { "El } \\
\text { independentismo } \\
\text { catalán es una } \\
\text { amenaza para los } \\
\text { ciudadanos } \\
\text { españoles". }\end{array}$ & Marco & $\begin{array}{l}\text { "Pedro Sánchez } \\
\text { es un traidor por } \\
\text { su asociación } \\
\text { con } \\
\text { independentistas } \\
\text { y etarras". } \\
\text { "El socialismo y } \\
\text { la izquierda son } \\
\text { enemigos de } \\
\text { España". }\end{array}$ & Marco & $\begin{array}{l}\text { "Pedro Sánchez } \\
\text { es un traidor } \\
\text { porque es } \\
\text { cómplice de los } \\
\text { nacionalistas y } \\
\text { separatistas". } \\
\text { "La alianza de } \\
\text { los enemigos de } \\
\text { España ha } \\
\text { creado una } \\
\text { emergencia } \\
\text { nacional". } \\
\text { "Los viejos } \\
\text { partidos (PSOE } \\
\text { y PP) no han } \\
\text { hecho } \\
\text { (absolutamente) } \\
\text { nada en los } \\
\text { pasados } 40 \\
\text { años". }\end{array}$ & Marco & $\begin{array}{l}\text { "Nosotros } \\
\text { defendemos } \\
\text { a los buenos } \\
\text { (los de } \\
\text { abajo) frente } \\
\text { a los malos } \\
\text { (los de } \\
\text { arriba)". } \\
\text { "Los viejos } \\
\text { partidos, el } \\
\text { PP y el } \\
\text { PSOE, no } \\
\text { cumplen con } \\
\text { lo que } \\
\text { prometen". }\end{array}$ \\
\hline
\end{tabular}

Fuente: elaboración propia.

\section{Discusión y conclusiones}

En las elecciones de abril de 2019, el crecimiento esperado de Ciudadanos, Podemos y Vox, opciones alternativas a PSOE y PP, los dos grandes partidos tradicionales, junto con la crispación creciente por la crisis política en Cataluña, influyeron en la construcción discursiva de bloques antagónicos y enfrentados: la izquierda versus la derecha, los "defensores de España" versus los "enemigos de España". También han contribuido a ello las apelaciones constantes a una supuesta relación del "otro" con comportamientos violentos. 
En el análisis de los dos debates, Pedro Sánchez del PSOE se centra en atacar a Casado (PP) y a Rivera (C'S); no ataca tanto a Iglesias (UP), con el que aún tenía la esperanza de pactar. Casado y Rivera, por su parte, dirigen las críticas más duras a Sánchez, que es realmente el objetivo a derribar, y algo menos, a Iglesias que, a su vez, utiliza un tono más moderado y define un enemigo, más bien colectivo, reflejado en los grandes poderes económicos relacionados con los políticos.

Para demostrar la superioridad de sus propuestas y vencer a sus adversarios en los debates y en las elecciones, los candidatos crean unos marcos que definen y delimitan quién es su enemigo, pero, sobre todo, quién es el enemigo de España y de los españoles. De acuerdo con el modelo de Lakoff, los políticos elaboran unos marcos con los que pretenden desmontar la propuesta política del "otro" y la trayectoria del partido, y demostrar la (in)capacidad del adversario para gobernar.

Cada uno se presenta a sí mismo y a su partido como la única alternativa para combatir al enemigo y defender a los "buenos", si ganan en la próxima contienda electoral. Los cuatro dejan claro, también, quién podría ser su posible aliado en los pactos poselectorales, y quién, no.

Todo ello queda reflejado en el uso que hacen del lenguaje, caracterizado por la entonación expresiva, el uso de marcadores, las figuras retóricas (la metáfora, la hipérbole, la ironía), la estructura de las frases, las enumeraciones, recursos todos ellos encaminados a transmitir con énfasis las ideas y los principales argumentos de los candidatos. Por otra parte, existen grandes coincidencias en los procedimientos utilizados por unos y otros, en general; en particular, Casado y Rivera crean los mismos marcos para presentar a Sánchez como enemigo principal del país. Del estudio realizado se deduce que coinciden en el uso de metáforas, estructuras sintácticas similares e, incluso, el uso del mismo verbo (romper), referido a España, como se recoge en los ejemplos (14) (Casado) y (28) y (29) (Rivera).

En conclusión, con el fin de crear y reforzar estas ideas en la mente del votante, los cuatro candidatos generan distintos tipos de argumentos, la mayoría de ellos, vacíos de contenido, pero con una fuerte carga emocional. A diferencia de lo que sucedía en los debates de los años 90, ahora lo que prima son las emociones. El ataque personal formulado a través del argumento ad personam, la elección del léxico con connotaciones negativas para referirse al rival (Iglesias: la gente no es idiota; Rivera: El Sr. Sánchez quiere tomar el pelo a los españoles), el uso de las figuras retóricas ya señalado, así como la estrategia descortés de la acusación de mentir, son los recursos utilizados para impresionar y convencer al electorado.

\section{Referencias bibliográficas}

Anaya, I. y Micovic, M. (2019). "Hasta aquí hemos llegado": análisis lingüístico y pragmático del debate entre Mariano Rajoy y Pedro Sánchez. En E. Conde-Vázquez, J. Fontenla-Pedreira \& J. Rúas-Araújo (Eds.), Debates electorales televisados: del antes al después. Cuadernos Artesanos de Comunicación, 154 (pp. 109-127). Sociedad Latina de Comunicación Social.

Arroyas Langa, E. y Pérez Díaz, P. L. (2016). La nueva narrativa identitaria del populismo: un análisis del discurso de Pablo Iglesias (Podemos) en Twitter. Cultura, lenguaje y representación: revista de estudios culturales de la Universitat Jaume I, (15), 51-63.

Benoit, W. L. (2002). The primary decision: A functional analysis of debates in presidential primaries. Greenwood Publishing Group. 
Blas Arroyo, J. L. (2011). Políticos en conflicto. Una aproximación pragmático-discursiva al debate electoral cara a cara. Peter Lang.

Cortés Rodríguez, L. (1991). Sobre conectores, expletivos y muletillas en el español hablado. Editorial Librería Ágora.

Cortés Rodríguez, L. (2008). Comentario pragmático de comunicación oral: Un discurso parlamentario. Arco Libros.

Fairclough, N. (1995). Critical Discourse Analysis. Addison Wesley.

Fernández García, F. (2017). La descortesía en el debate electoral cara a cara. Universidad de Sevilla.

Fernández Lagunilla, M. (1990). La lengua en la comunicación politica, vol. I (El discurso del poder). Arco/Libros.

Fuentes Rodríguez, C. (2010). El debate entre Zapatero/Rajoy: estudio argumentativo. Tonos Digital, (20). http://www.um.es/tonosdigital/znum20/secciones/estudios-6-el_debate entre_z_y_r_2.htm

García Beaudoux, V., D’Adamo, O. y Slavinsky, G. (2005). Comunicación política y campañas electorales. Gedisa.

Goffman, E. (1974). Frame analysis: An essay on the organization of experience. Harper and Row.

Lakoff, G. (2004). No pienses en un elefante: Lenguaje y debate político. Ed. Complutense, 2006.

Lakoff, G. (2009). The political mind: a cognitive scientist's guide to your brain and its politics. Penguin Books.

Micovic, M. (2014). La comunicación y el discurso políticos en España y Serbia. Análisis comparativo de las estrategias argumentativas utilizadas en los debates electorales televisivos. (Tesis doctoral). http://diposit.ub.edu/dspace/handle/2445/62703

Montolío Durán, E. (2001). Conectores de la lengua escrita. Ariel.

Montolío Durán, E. (2019). Tomar la palabra. Política, género y nuevas tecnologías en la comunicación. Edicions de la Universitat de Barcelona.

Morales López, E. y Prego Vázquez, G. (2002). Entrevistas electorales en las campañas políticas para la presidencia del gobierno de 1996 y 2000. Oralia, (5), 203-245.

Perelman, Ch. y Olbrechts-Tyteca, L. (1958). Tratado de la argumentación. La nueva retórica. Gredos, 2000.

Reinemann, C., Aalberg, T., Esser, F., Strömbäck, J., \& de Vreese, C. H. (2016). Populist political communication: Toward a model of its causes, forms, and effects. En T. Aalberg, F. Esser, C. Reinemann, J. Strömbäck, \& C. H. de Vreese (Eds.), Populist political communication in Europe (pp. 22-36). Routlege. 
Leanne, S. (2009). Hablar como Obama. Profit Editorial.

Solanilla, P. (2019). La crisis del lenguaje de la política. La revista de ACOP, (40), 7-11. https://compolitica.com/wpcontent/uploads/2019/07/N40_Eta.2_La_revista_de_ACOP_Julio2019I.pdf

Van Dijk, T. A. (2005). Política, ideología y discurso. Quórum Académico, 2(2), 15-47.

Van Dijk, T. A. (2008). Discurso y Poder. Gedisa.

Wodak, R. (2000). Discourse and Discrimination: Rhetorics of Racism and Antisemitism. Routledge.

\section{AUTORES:}

\section{Miljana Micovic}

Doctora por la Universitat de Barcelona en el campo de la comunicación y discurso políticos. Es Jefa de Estudios y docente del Centro de Educación Superior Next en Madrid (España). Trabajó durante siete años como Coordinadora Académica y docente de Next International Business School en Madrid. Ha sido profesora del debate y discurso político invitada en numerosos másteres en España. Es miembro del Consejo Directivo de ACOP (Asociación de Comunicación Política), forma parte del grupo de investigación EDAP (Estudios del Discurso Académico y Profesional) como miembro colaborador profesional. Su línea de investigación está centrada en el análisis del discurso político, en general, y en los debates electorales, en concreto. Su índice H es 1 (diciembre 2019).

m.micovic@cesnext.com

\section{Índice $\mathrm{H}: 1$}

Orcid ID: https://orcid.org/0000-0002-1944-6446

Google Scholar: https://scholar.google.es/citations?user=MXtTY9kAAAAJ\&hl=es\&oi=ao

\section{Adrià Alsina Leal}

Doctorando en Comunicación Política por la Universidad Central de Catalunya (UVic - UCC) y profesor asociado de Periodismo Internacional y Estrategias de Comunicación Global en esta universidad. También ha dado clases en la Universidad China de Hong Kong y en La Sorbonne de París. Empezó su carrera profesional como periodista para varios medios de Barcelona, incluyendo la cadena de televisión pública TV3. Ha trabajado como corresponsal en Barcelona para medios como Postimees de Estonia. Ha sido consultor de comunicación del Grupo IFC-Banco Mundial, del Banco Interamericano de Desarrollo (BID), el Banco Africano de Desarrollo (AfDB y la Organización para la Cooperación) y el Desarrollo Económico (OCDE). Fue jefe de comunicación de la Asamblea Nacional Catalana (ANC) entre 2016 y 2018. Su índice H es 0 (diciembre 2019).

adria.alsina@uvic.cat

Orcid ID: https://orcid.org/0000-0002-3984-9453

\section{Inmaculada Anaya Revuelta}

Doctora y Premio Extraordinario por la Universidad Complutense de Madrid. Profesora Titular de Lengua Española en la Universidad de Vigo desde 1999. Desde noviembre de 2015, vicedecana de Organización académica. Dirigió Evágora, asesoría de comunicación para empresas e instituciones. En el año 2004 es nombrada Socia colaboradora del Círculo de Empresarios de Galicia (CFV). Dirigió y coordinó el Máster universitario en Lengua y Comunicación en los negocios. Imparte clases en el Departamento de Lengua Española en distintas titulaciones. Ha publicado en distintas revistas de alto impacto estudios centrados en el análisis del discurso y en diversos aspectos de la 
lengua. Desarrolla una amplia labor en la formación para empresas e instituciones como experta en comunicación. En los últimos 10 años ha impartido cursos y talleres, entre otras, en las siguientes organizaciones: Instituto de Comunicación Empresarial (ICE); P\&A Consultoría y Formación S. L; Escuela de Negocios de Vigo; ALDESA (Madrid); FENOSA (Bilbao), TSOLAR (Madrid), etc. Participa como investigadora en el proyecto "Debates electorales televisados en España: modelos, proceso, diagnóstico y respuesta", financiado por el Ministerio de Economía, Industria y Competitividad. Su índice H es 4 (diciembre 2019).

ianaya@uvigo.es

Índice H: 4

Orcid ID: https://orcid.org/0000-0002-1883-4922

Google Scholar: $\underline{\text { https://scholar.google.es/citations?user=r88j4V0AAAAJ\&hl=es\&oi=ao }}$ 